\title{
Adaptive Fixed-Time Stability Control and Parameters Identification for Chaotic Oscillation in Second Order Power System
}

\author{
Caoyuan Ma $\mathbb{D}^{1,2}$ Wenbei Wu $\mathbb{D}^{1,2}$ Zhijie Li $\mathbb{D}^{1,2}$ Yuzhou Cheng $\mathbb{D},{ }^{1,2}$ and Faxin Wang $\mathbb{D}^{1,2}$ \\ ${ }^{1}$ Jiangsu Province Laboratory of Mining Electric and Automation, China University of Mining and Technology, 221116, China \\ ${ }^{2}$ School of Electrical and Power Engineering, China University of Mining and Technology, Xuzhou 221008, China \\ Correspondence should be addressed to Yuzhou Cheng; 836754437@qq.com
}

Received 11 July 2018; Revised 28 October 2018; Accepted 14 November 2018; Published 29 November 2018

Academic Editor: Dragan Poljak

Copyright (C) 2018 Caoyuan Ma et al. This is an open access article distributed under the Creative Commons Attribution License, which permits unrestricted use, distribution, and reproduction in any medium, provided the original work is properly cited.

In this paper, the novel adaptive fixed-time stability control for chaotic oscillation in second order power system is proposed. The settling time of fixed-time control can be adjusted to the desired value without knowing the initial condition, while the finite time control depends on that. Then, we develop a parameter identification method of fixed-time depending on synchronous observer with adaptive law of parameters, which can guarantee these uncertain parameters to be identified effectively. Finally, some numerical results demonstrate the effectiveness and practicability of the scheme.

\section{Introduction}

Over the past few decades, the mechanism of bifurcation and chaos in power system has been intensively studied. In 1980, Kopell [1] first studied how to turn a three-machine power system into a two-degree-of-freedom system and analyzed the bifurcation and chaos of the system by Meilenikov method, which marked the beginning of chaos research in power system. In 1990, American scientists Ott, Grebogi, and Yorke [2] used the OGY method to achieve the true sense of chaos control. In [3], Wang carried out chaotic control on a simplified second order model of power system and adopted the classical backstepping control algorithm to control the chaotic power system to a stable flat point. At present, many literatures have made theoretical analyses of chaos control, while the study of chaotic oscillation control strategy in power system has just started.

Power system, as a typical nonlinear and nonautonomous system, contains many complex nonlinear electromechanical oscillations such as low frequency oscillation, subsynchronous oscillation, bifurcation, and chaos oscillation [4-6]. When periodic load disturbance reaches a certain amplitude, chaos oscillation phenomena in power system will happen, which is an aperiodic, irregularity, paroxysmal and sudden ill-conditioned electromechanical chaotic oscillation $[7,8]$. In serious cases, chaos oscillation will lead to interconnection of power system and a serious threat to the safety of the power grid [9].

In recent years, with the development of the nonlinear system control theory, some useful control methods have been widely used in power system. In [10], a kind of adaptivefeedback control method was proposed to control the chaos in power system, which systematize and structure the design process of the system's Lyapunov functions and controllers through the reverse design. But its structure is very complex, and the complexity of regression matrix would become stronger especially when the nonlinear damping existed for the system parameters uncertainty. In [11], the authors discussed the fuzzy control of chaotic power system with uncertainly. However, it needs to adjust the control rules and parameters constantly, which affect dynamic response and steady state precision. In [12], Zhao designed a fuzzy sliding mode variable structure controller, which is robust to disturbances, maintaining the advantages of fuzzy control and sliding mode control. In [13], by using the sliding mode control, a nonsingular terminal sliding-mode controller with 
nonlinear disturbance observer was proposed for chaotic oscillation in power system, which can shorten the reaching time and weaken system chattering. However, these methods cannot guarantee stability in a certain period of time and cannot guarantee convergence of speed error responses under uncertain parameters. Recently, the finite-time control and synchronization of the chaotic power system have attracted interests of many researchers [14-18]. In [19], the authors studied the chaos control of power system based on the finite-time stability theory. In [20], an adaptive finite-time stability controller was presented for chaotic power system with uncertain parameters.

Although the finite-time control is better than those described above in control effect, the convergence rate of the finite-time control is extremely dependent on the initial conditions. In the power system, the initial conditions can hardly be given and estimated, which may result in different convergence time and lead to the deterioration of the system's performance. To solve this problem, Polyakov [21] first proposed the definition of fixed-time stability. The settling time of fixed-time control can be adjusted to the desired value without knowing the initial condition. Subsequently, fixed time control began to be used in some complex systems and showed good performance [22-26].

According to the discussion of the above, the paper presents an adaptive fixed-time control strategy which can accelerate the convergence time, suppress chaos in the power system, and avoid voltage collapse. The main advantages of the proposed controller are that it can guarantee the system stable in fixed time without depending on initial state and the settling time can be calculated directly. This paper first discusses the use of adaptive fixed-time stability analysis with uncertain parameters for chaotic oscillation in second order power system. The structure of this paper is arranged as follows. In Section 2, we introduce the model of chaotic oscillation in second order power system, some useful definitions and lemmas. In Section 3, the novel adaptive fixedtime stability control for chaotic oscillation in second order power system is proposed, and some numerical examples are provided to demonstrate the effectiveness and practicability of the results. Section 4 proposes a parameter identification method of fixed-time depending on synchronous observer with adaptive law of parameters. At last, we make some conclusions.

\section{Model Description}

Without the influence of the internal factors such as the moment of inertia of the equivalent system, the dimensionless mathematical model of chaotic oscillation in second order power system is considered [27]:

$$
\begin{aligned}
\dot{\sigma}= & \omega(t) \\
\dot{\omega}(t)= & -\frac{P_{\max }}{H} \sin \sigma(t)-\frac{D}{H} \omega(t)+\frac{P_{m}}{H}+\frac{P_{\xi}}{H} \cos f t \\
& -\frac{\delta}{H} \cos z t \sin \sigma(t)
\end{aligned}
$$

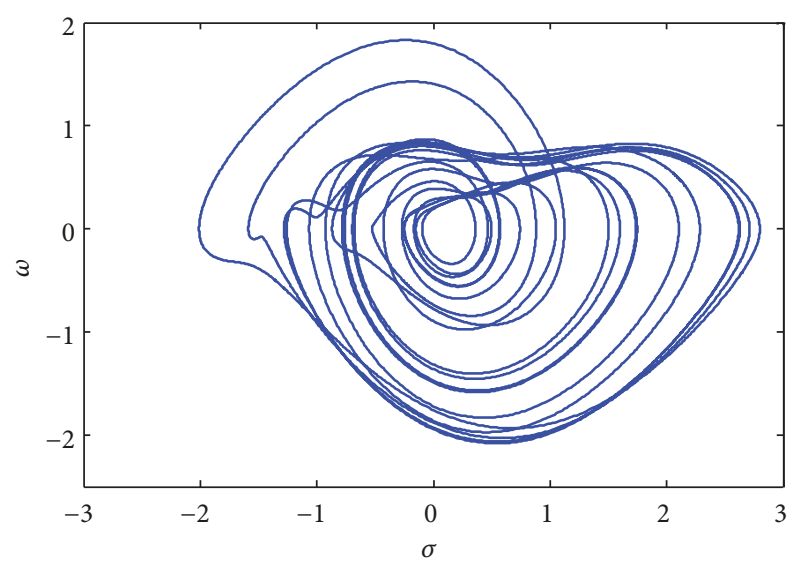

FIGURE 1: Chaotic attractor in $(x, y)$-space.

where $\sigma(t)=\sigma_{1}-\sigma_{2}$ is the relative angle between the system (1) equivalent generator and the system 2 equivalent generator $\mathrm{q}$ axis potential, rad; $H$ is equivalent moment of inertia, $\mathrm{kg} \cdot \mathrm{m}^{2} ; \omega(t)$ is relative angular velocity, $\mathrm{rad} / \mathrm{s} ; D$ is equivalent damping coefficient, $N \cdot m \cdot s / \mathrm{rad} ; P_{m}$ is the mechanical power of the equivalent generator $1, W ; P_{\xi}$ is disturbance power amplitude, $W ; \delta=\Delta p P_{\max }$ is electromagnetic power perturbation amplitude, and $f$ is the frequency of the disturbance power, $H z ; \Delta p$ is increased electromagnetic power. In this case, $P_{\xi}$ and $\delta$ are the perturbation amplitudes of the two perturbed terms in the system.

The bifurcation and chaos phenomena of the above system (1) are fully investigated in Refs [28]. For example, if given $z=0.8 \mathrm{~Hz}, P_{\xi}=2 \mathrm{~W}, P_{\max }=100 \mathrm{~W}, \mathrm{H}=100 \mathrm{~kg} \cdot \mathrm{m}^{2}$, $D=40 \mathrm{~N} \cdot \mathrm{m} \cdot \mathrm{s} / \mathrm{rad}, f=1 \mathrm{~Hz}, \delta \geq 127$, the chaotic behavior can be found in Figure 1.

The chaotic oscillation of the power system poses a serious threat to the safe operation of the power grid. Therefore, finding an appropriate control method to solve this problem is significant. In this paper, we eliminate the chaos in secondorder chaotic oscillation system by employing the adaptive fixed-time controller. The following definition and lemma are introduced in advance, which are required for proving main results.

Definition 1 (see [21]). Consider the following dynamic system

$$
\dot{x}=f(x)
$$

where $x \in R^{n}$ is the system state, and $f$ is a smooth nonlinear function. If, for any initial condition, there exists a fixed settling time $T_{0}$, which is not connected with the initial condition, such that

$$
\lim _{t \longrightarrow T_{0}}\|x(t)\|=0
$$

and $\|x(t)\| \equiv 0$, if $t \geq T_{0}$, then the system (2) is said to be fixed-time stable.

Lemma 2 (see [29]). Suppose there exists a continuous function $V(t):[0, \infty) \longrightarrow[0, \infty)$ such that 
(1) $V$ is positive definite.

(2) There exist real numbers $c>0$ and $0<\rho<1$ such that

$$
V^{\dot{\prime}}(t) \leq-c V^{\rho}(t), \quad t \geq t_{0}
$$

then one has

$$
V^{1-\rho}(t) \leq V^{1-\rho}\left(t_{0}\right)-c(1-\rho)\left(t-t_{0}\right), \quad t_{0} \leq t \leq t^{*}
$$

and

$$
V(t)=0, \quad t \geq t^{*}
$$

of which

$$
t^{*}=t_{0}+\frac{V^{1-\rho}\left(t_{0}\right)}{c(1-\rho)} .
$$

Lemma 3 (see [21]). If there exists a continuous radically unbounded function $V: R^{N} \longrightarrow R_{+} \cup|0|$ such that

(1) $V(x)=0 \Longleftrightarrow x=0$;

(2) any solution $x(t)$ satisfied the inequality $D * V(x(t)) \leq$ $-\left[\alpha V^{p}(x(t))+\beta V^{q}(x(t))\right]^{k}$ for some $\alpha, \beta, p, q, k>$ 0 , pk $<1$, and $q k>1$, where $D * V(x(t))$ denotes the upper right hand derivative of the function $V(x(t))$;

then the origin is globally fixed time stable and the following estimate holds:

$$
T\left(x_{0}\right) \leq \frac{1}{\alpha^{k}(1-p k)}+\frac{1}{\beta^{k}(q k-1)}, \quad \forall x_{0} \in R^{n} .
$$

Lemma 3 presents quite a conservative settling time estimate. A more accurate estimate is provided in the next lemma. Consider the case where constants $\mathrm{p}$ and $\mathrm{q}$ are of the form $p=1-1 / 2 \gamma$ and $q=1+1 / 2 \gamma, \gamma>1$.

Lemma 4 (see [30]). If $x_{1}, x_{2} \ldots x_{N} \geq 0$, then

$$
\begin{aligned}
& \sum_{i=1}^{N} x_{i}^{\eta} \geq\left(\sum_{i=1}^{N} x_{i}\right)^{\eta}, \quad 0<\eta \leq 1, \\
& \sum_{i=1}^{N} x_{i}^{\varsigma} \geq N^{1-\varsigma}\left(\sum_{i=1}^{N} x_{i}\right)^{\varsigma}, \quad \varsigma>1 .
\end{aligned}
$$

\section{Main Results}

\subsection{Fixed-Time Chaotic Oscillation Control of Power System}

Theorem 5. The fixed-time stability of chaotic oscillation in second order power system can be achieved by adding the following controller $u_{1}$

$$
\begin{aligned}
u_{1}= & \frac{P_{\max }}{H} \sin \sigma-\frac{P_{m}}{H}-\frac{P_{\xi}}{H} \cos f t+\frac{\delta}{H} \cos z t \sin \sigma \\
& -k_{1} \operatorname{sign}(\omega)|\omega|^{\alpha}-k_{1} \omega^{\beta}
\end{aligned}
$$

where $0<\alpha<1, \beta>1, k_{1}>0, k_{1}$ is the tuning parameter feedback gain of the terminal attractor, and

$$
\dot{k_{1}}=|\omega|^{\alpha+1}+\omega^{\beta+1}-\left(k_{1}-g_{1}\right)^{\alpha}-\left(k_{1}-g_{1}\right)^{\beta}
$$

where $g$ is the arbitrary positive constant.
The system is described as

$$
\begin{aligned}
\dot{\sigma}= & \omega(t) \\
\dot{\omega}(t)= & -\frac{P_{\max }}{H} \sin \sigma(t)-\frac{D}{H} \omega(t)+\frac{P_{m}}{H}+\frac{P_{\xi}}{H} \cos f t \\
& -\frac{\delta}{H} \cos z t \sin \sigma(t)+u_{1}
\end{aligned}
$$

Proof. For analysis convenience, we select the Lyapunov candidate function

$$
V_{1}(t)=\frac{1}{2} \omega^{2}+\frac{1}{2}\left(k_{1}-g_{1}\right)^{2}
$$

we can get the derivative of the system trajectory, by using the design of the controller $u_{1}$ and the corresponding tuning parameters.

$$
\left.\begin{array}{rl}
\dot{V}_{1}(t) & =\omega \dot{\omega}+\left(k_{1}-g_{1}\right) \dot{k_{1}} \\
= & -\frac{D}{H} \omega^{2}-g_{1}|\omega|^{\alpha+1}-g_{1} \omega^{\beta+1}-\left(k_{1}-g_{1}\right)^{\alpha+1} \\
& -\left(k_{1}-g_{1}\right)^{\beta+1} \\
\leq & -g_{1}|\omega|^{\alpha+1}-g_{1} \omega^{\beta+1}-\left(k_{1}-g_{1}\right)^{\alpha+1} \\
& -\left(k_{1}-g_{1}\right)^{\beta+1} \\
= & -2^{(1 / 2)(\alpha+1)} g_{1}\left(\frac{1}{2} \omega^{2}\right)^{(1 / 2)(\alpha+1)} \\
& -2^{(1 / 2)(\alpha+1)}\left[\frac{1}{2}\left(k_{1}-g_{1}\right)^{2}\right]^{(1 / 2)(\alpha+1)} \\
& -2^{(1 / 2)(\beta+1)} g_{1}\left(\frac{1}{2} \omega^{2}\right)^{(1 / 2)(\beta+1)} \\
& -2^{(1 / 2)(\beta+1)}\left[\frac{1}{2}\left(k_{1}-g_{1}\right)^{2}\right]^{(1 / 2)(\beta+1)} \\
\leq & -m_{1}\left\{\left(\frac{1}{2} \omega^{2}\right)^{(1 / 2)(\alpha+1)}+\left[\frac{1}{2}\left(k_{1}-g_{1}\right)^{2}\right]^{(1 / 2)(\alpha+1)}\right. \\
\left\{\frac{1}{2}\left(k_{1}-g_{1}\right)^{2}\right]^{(1 / 2)(\beta+1)}
\end{array}\right\}
$$

where $m_{1}=\min \left\{2^{(1 / 2)(\alpha+1)} g_{1}, 2^{(1 / 2)(\alpha+1)}\right\}, \quad n_{1}=$ $\min \left\{2^{(1 / 2)(\beta+1)} g_{1}, 2^{(1 / 2)(\beta+1)}\right\}$

Thus, it follows from Lemma 4 that

$$
\begin{aligned}
\dot{V}_{1}(t) & \\
\leq & -m_{1}\left\{\left(\frac{1}{2} \omega^{2}\right)+\left[\frac{1}{2}\left(k_{1}-g_{1}\right)^{2}\right]\right\}^{(1 / 2)(\alpha+1)} \\
& -2^{(1-\beta) / 2} n_{1}\left\{\left(\frac{1}{2} \omega^{2}\right)+\left[\frac{1}{2}\left(k_{1}-g_{1}\right)^{2}\right]\right\}^{(1 / 2)(\beta+1)} \\
= & -m_{1} V_{1}^{(1 / 2)(\alpha+1)}-2^{(1-\beta) / 2} n_{1} V_{1}^{(1 / 2)(\beta+1)}
\end{aligned}
$$

Thus, it follows from Lemma 2 , where $0<\alpha<1, \beta>1$. 


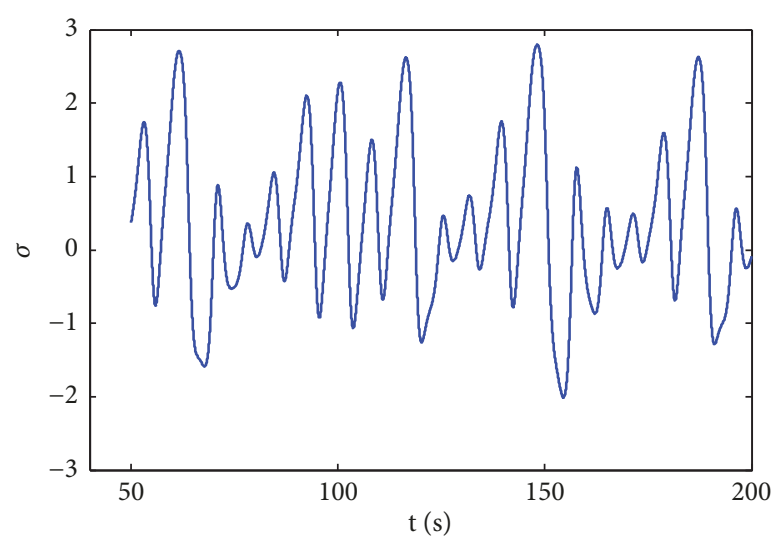

(a)

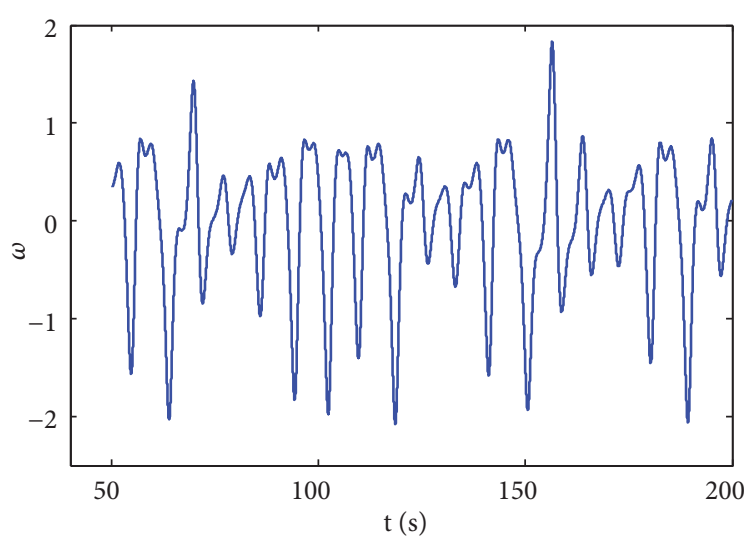

(b)

Figure 2: The chaotic state trajectory of the system (1) without control. (a) The timing diagram of $\sigma$. (b) The timing diagram of $\omega$.

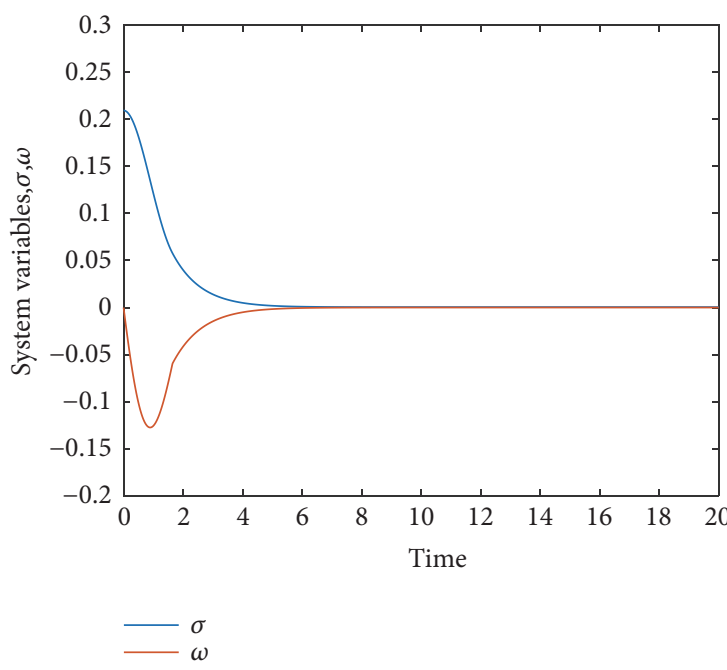

(a)

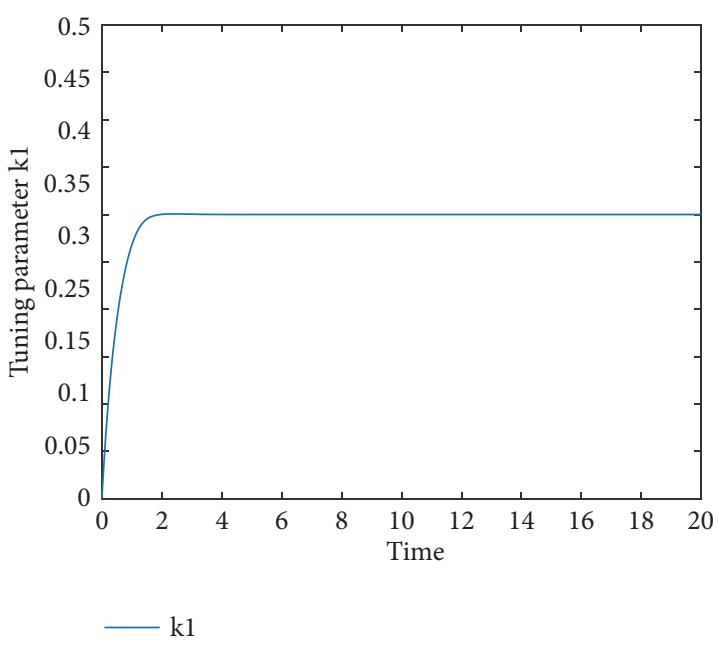

(b)

Figure 3: (a) The evolution of the controlled state variables $\sigma$ and $\omega$. (b) Tuning parameter $k_{1}$.

The system (12) will be stable in fixed time $T_{1}$, where

$$
\begin{aligned}
T_{1} \leq & \frac{1}{m_{1}(1-(\alpha+1) / 2)} \\
& +\frac{1}{2^{(1-\beta) / 2} n_{1}((\beta+1) / 2-1)} \\
= & \frac{2}{m_{1}(1-\alpha)}+\frac{2^{(\beta+1) / 2}}{n_{1}(\beta-1)}
\end{aligned}
$$

when $t \geq T_{1}, \omega \equiv 0, k_{1}=g_{1}$. Therefore, based on the adaptive control strategy, the chaotic system will be stable for a fixed time.

3.2. Numerical Examples. In this section, numerical results are performed to demonstrate the effectiveness and practicability of the scheme. We give $z=0.8 \mathrm{~Hz}, P_{\xi}=2 \mathrm{~W}, P_{\max }=$ $100 \mathrm{~W}, H=100 \mathrm{~kg} \cdot \mathrm{m}^{2}, D=40 \mathrm{~N} \cdot \mathrm{m} \cdot \mathrm{s} / \mathrm{rad}, f=1 \mathrm{~Hz}, \alpha=0.8$, $\beta=1.1, k_{1}(0)=0$, and the initial value of the variables is to be $\{\sigma, \omega\}=\{\pi / 15,0\}$.

Figures 2(a) and 2(b) are the response chaotic trajectories of the system variables $\sigma$ and $\omega$ when the second order chaotic oscillation system is not controlled. From Figure 2, it is clear that the state of the system variables $\omega$ and $\sigma$ is aperiodic and is always in a state of instability.

Figure 3(a) is the response curves of the system variables $\sigma$ and $\omega$ after the fixed-time controllers $u_{1}$ are applied to the second order chaotic oscillation system. Figure 3 (b) shows the evolution of tuning parameter of terminal attractors $k_{1}$. From Figures 2 and $3(\mathrm{a})$, it is clear that when the system is chaotic before $1 \mathrm{~s}$ without the controller, once the controller is applied, the variables $\sigma$ and $\omega$ become stable after transition process $4.2 \mathrm{~s}$, simultaneously. The adaptive fixedtime controller synchronizes the chaotic system to a stable state without chaos, and the system eventually converges to the origin. Thus, the simulation results show that the designed controller is feasible and achieves the desired effect. 


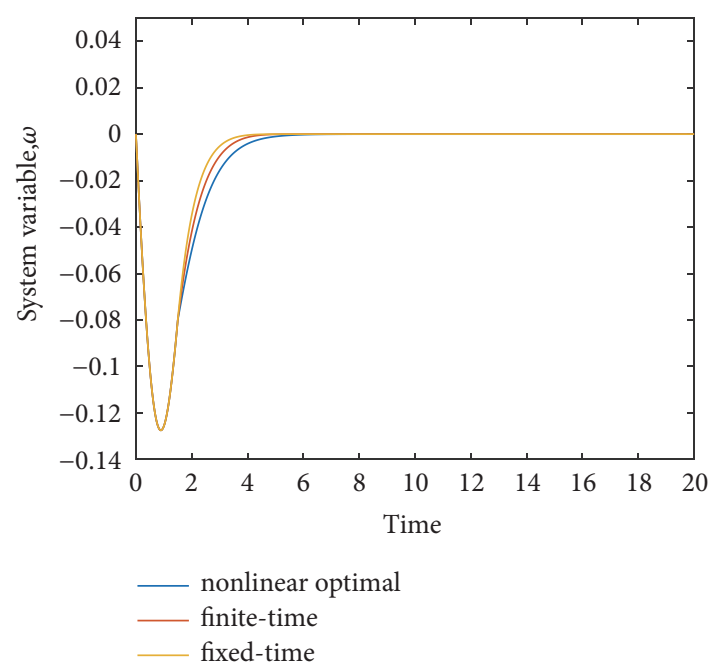

Figure 4: Comparison of dynamic response of different control modes with $\alpha=0.8$ and $\beta=1.1$.

To ensure a fair comparison with other control methods, we set constant initial conditions and tuning parameter $\mathrm{f}$ the terminal attractor $k_{1}$ and investigated $\omega$ convergence for fixed-time control, finite-time control, and nonlinear optimal control numerically. As shown in Figure 4, convergence time for the proposed fixed-time controller is significantly less than that for the other controllers. The system state variable $\omega$ achieves stable state at $4.2 \mathrm{~s}$ under the action of fixed-time controllers. However, the system state variable $\omega$ achieves stable state at $5 \mathrm{~s}$ and $5.3 \mathrm{~s}$ under the action of finite-time controller and nonlinear optimal control, respectively. Thus, the fixed-time method has the better capacity to handle a nonlinear system in a short time.

To explore the relationship between the convergence time and the values of the parameters $\alpha$ and $\beta$ experimentally, we select system state variable $\omega$ to demonstrate the convergence time. Figures 5(a) and 5(b) are, respectively, convergence for $\omega$ that increases with increasing $\alpha$ and $\beta$ parameter values under the fixed-time controller. In Figure 5(a), the parameter values are $\beta=1.8$ and $\alpha=0.3,0.5,0.7,0.9$. In Figure 5(b), the parameter values are $\alpha=0.8$ and $\beta=1.1,1.3,1.5,1.7$. In Figures 5(a) and 5(b), the system parameters and other controller parameters and tuning parameters of the terminal attractor are consistent with the previous sections. The simulation results clearly show that changing the controller parameters $\alpha$ and $\beta$ can change the time of the system state variable $\omega$ to reach the steady state. And the smaller the $\alpha$ and $\beta$ values of the system are, the faster the convergence time will be. Moreover, the influence of $\alpha$ on the convergence time of the system state variable $\omega$ is more than the influence of $\beta$ on it. The simulation results are consistent with the theoretical analysis of the maximum stable time $T_{1}$ of the system in the previous section. Thus, the values of $\alpha$ and $\beta$ also affect the stability value of the system state That is to say, we can get the size of the system state variables to the numerical value we need by controlling the size of $\alpha$ and $\beta$.

\section{Fixed-Time Synchronization and Parameters Identification}

In the long-term operation of the grid, changes in the working environment will affect the parameters of the grid model. Therefore, it is very important to identify the parameters of the chaotic model of the power grid. Without considering the effects of higher harmonic disturbances, electromagnetic interference, etc., according to the previous analysis, the uncertain response system is given as follows:

$$
\begin{aligned}
\dot{\sigma}(t)= & \omega(t) \\
\dot{\omega}(t)= & -\frac{P_{\max }}{H} \sin \sigma(t)-\frac{D}{H} \omega(t)+\frac{P_{m}}{H} \\
& -\frac{\delta}{H} \cos z t \sin \sigma(t) \\
= & a \sin \sigma-b \omega+c-d \cos z t \sin \sigma
\end{aligned}
$$

The following describes the identification of unknown parameters in the model.

Theorem 6. Construct a synchronization parameter observer [31]

$$
\dot{\hat{\omega}}=\widehat{a} \sin \sigma-\widehat{b} \omega+\widehat{c}-\widehat{d} \cos z t \sin \sigma+u_{2}
$$

Take the controller:

$$
u_{2}=-k_{2}^{2} e-k_{2} \operatorname{sign}(e)|e|^{\alpha}-k_{2} \operatorname{sign}(e)|e|^{\beta}
$$

and the following adaptive law of parameters:

$$
\begin{aligned}
& \dot{\hat{a}}=-h_{1} e \sin x \\
& \dot{\vec{b}}=-h_{2} e y \\
& \dot{\hat{c}}=-h_{3} e \\
& \dot{\hat{d}}=-h_{4} e \cos z t \sin x
\end{aligned}
$$

where $e=\widehat{\omega}-\omega$ is the synchronization error between the observer state variable and the original system state variable; $h_{i}(i=1,2,3,4)$ is the arbitrary positive constant; $\dot{k}_{2}=|e|^{\alpha+1}+$ $|e|^{\beta+1}-\left(k_{2}-g_{2}\right)^{\alpha}-\left(k_{2}-g_{2}\right)^{\beta}$ is tuning parameter feedback gain, and $g_{2}$ is the arbitrary positive constant. The meaning of " $y$ " is the system state variable $\omega$, the meaning of " $x$ " is the system state variable $\sigma$. Then the state variables in the observer (18) can be synchronized with the state variables in the system (17), and the unknown parameters $a, b, c, d$ can be identified.

Proof. The error system can be obtained from (18)-(20):

$$
\begin{aligned}
\dot{e} & =e_{1} \sin x+e_{2} y+e_{3}+e_{4} \cos z t \sin x+u_{2} \\
\dot{e_{1}} & =-h_{1} e \sin x \\
\dot{e_{2}} & =-h_{2} e y \\
\dot{e_{3}} & =-h_{3} e \\
\dot{e_{4}} & =-h_{4} e \cos z t \sin x
\end{aligned}
$$




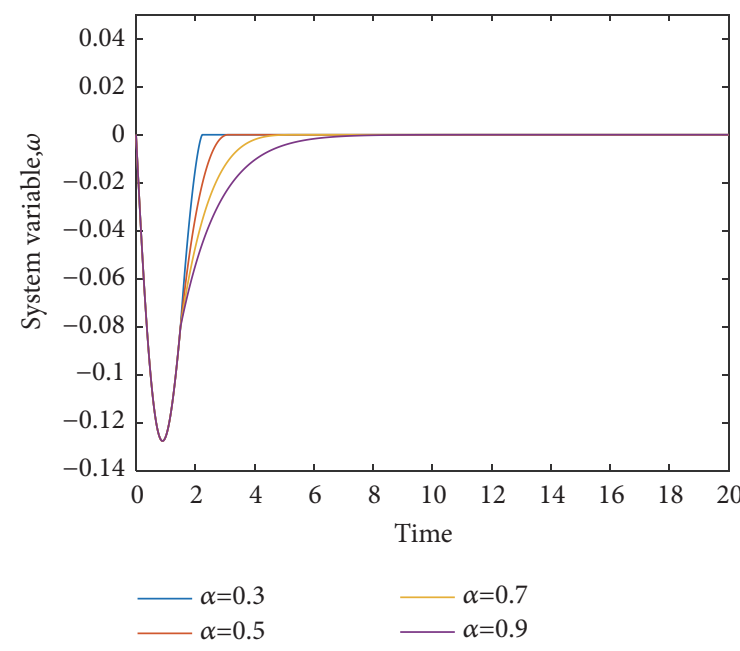

(a)

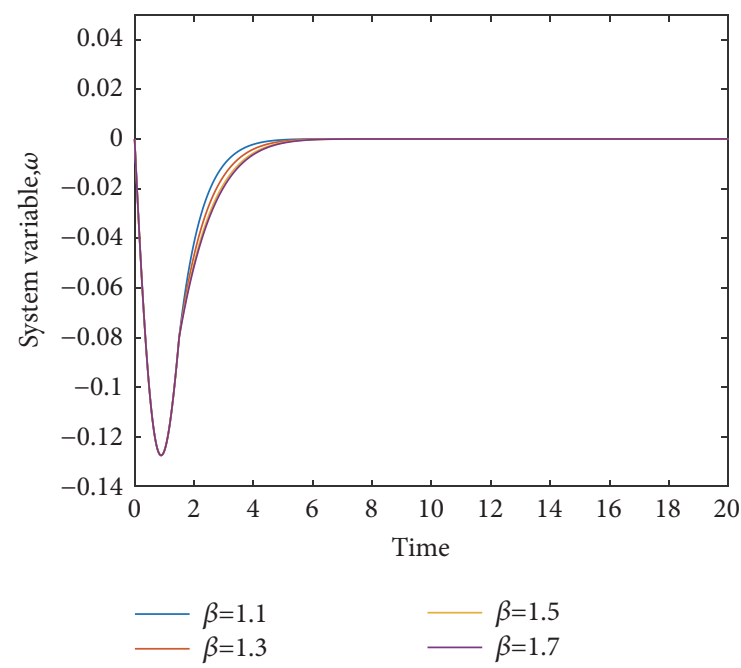

(b)

FIGURE 5: The effect of the control parameter on the response speed of the variable $\omega$. (a) The variations of $\omega$ with $\beta=1.8$ and $\alpha=$ $0.3,0.5,0.7,0.9$. (b) The variations of $\omega$ with $\alpha=0.8$ and $\beta=1.1,1.3,1.5,1.7$.

where $e_{j}=(j=1,2,3,4)$ is the error between the estimated and the actual value of the unknown parameter.

We select the Lyapunov candidate function

$$
V_{2}(t)=\frac{1}{2} e^{2}+\sum_{j=1}^{4} \frac{1}{2 h_{j}} e_{j}^{2}+\frac{1}{2}\left(k_{2}-g_{2}\right)^{2}
$$

The derivative of the subsystem trajectory in (22) can be obtained:

$$
\begin{aligned}
& \dot{V}_{2}(t)=e \dot{e}+\sum_{j=1}^{4} \frac{1}{h_{j}} e_{j} \dot{e}_{j}+\left(k_{2}-g_{2}\right) \dot{k_{2}}=e\left(e_{1} \sin x\right. \\
& +e_{2} y+e_{3}+e_{4} \cos z t \sin x+k_{2}^{2} e-k_{2} \operatorname{sign}(e)|e|^{\alpha} \\
& \left.-k_{2} \operatorname{sign}(e)|e|^{\beta}\right)+\frac{1}{h_{1}} e_{1}\left(-h_{1} e \sin x\right)+\frac{1}{h_{2}} \\
& \cdot e_{2}\left(-h_{2} e y\right)+\frac{1}{h_{3}} e_{3}\left(-h_{3} e\right)+\frac{1}{h_{4}} \\
& \cdot e_{4}\left(-h_{4} e \cos z t \sin x\right)+\left(k_{2}-g_{2}\right)\left[|e|^{\alpha+1}+|e|^{\beta+1}\right. \\
& \left.-\left(k_{2}-g_{2}\right)^{\alpha}-\left(k_{2}-g_{2}\right)^{\beta}\right] \leq-g_{2}|e|^{\alpha+1} \\
& -g_{2}|e|^{\beta+1}-\left(k_{2}-g_{2}\right)^{\alpha+1}-\left(k_{2}-g_{2}\right)^{\beta+1} \\
& \quad \leq-m_{2}\left\{\left(\frac{1}{2} e^{2}\right)^{(1 / 2)(\alpha+1)}\right\}-n_{2}\left\{\left(\frac{1}{2} e^{2}\right)^{(1 / 2)(\beta+1)}\right. \\
& \left.+\left[\frac{1}{2}\left(k_{2}-g_{2}\right)^{2}\right]^{(1 / 2)(\alpha+1)}\right\} \\
& +[1 / 2)(\beta+1) \\
& +
\end{aligned}
$$

where $m_{2}=\min \left\{2^{(1 / 2)(\alpha+1)} g_{2}, 2^{(1 / 2)(\alpha+1)}\right\}, \quad n_{2}=$ $\min \left\{2^{(1 / 2)(\beta+1)} g_{2}, 2^{(1 / 2)(\beta+1)}\right\}$ Thus, it follows from Lemma 4 that

$$
\begin{aligned}
\dot{V}_{2}(t) & \\
\leq & -m_{2}\left\{\left(\frac{1}{2} e^{2}\right)+\left[\frac{1}{2}\left(k_{2}-g_{2}\right)^{2}\right]\right\}^{(1 / 2)(\alpha+1)} \\
& -2^{(1-\beta) / 2} n_{2}\left\{\left(\frac{1}{2} e^{2}\right)+\left[\frac{1}{2}\left(k_{2}-g_{2}\right)^{2}\right]\right\}^{(1 / 2)(\beta+1)} \\
= & -m_{2} V_{2}^{(1 / 2)(\alpha+1)}-2^{(1-\beta) / 2} n_{2} V_{2}^{(1 / 2)(\beta+1)}
\end{aligned}
$$

We can obtain stable time of the error system (21) by Lemma 3:

$$
\begin{aligned}
T_{2} \leq & \frac{1}{m_{2}(1-(\alpha+1) / 2)} \\
& +\frac{1}{2^{(1-\beta) / 2} n_{2}((\beta+1) / 2-1)} \\
= & \frac{2}{m_{2}(1-\alpha)}+\frac{2^{(\beta+1) / 2}}{n_{2}(\beta-1)}
\end{aligned}
$$

which means that $e \equiv 0, k_{2}=g_{2}, \widehat{a}=a, \widehat{b}=b, \widehat{c}=c, \widehat{d}=d$ when $t \geq T_{2}$.

Then, we compare the parameter identification method based on the fixed-time synchronization observer with the parameter identification method which did not introduce the fixed-time control. If given $\sigma=\pi / 15, \omega=0, h_{1}=h_{2}=$ $h_{3}=h_{4}=50, \alpha=0.8, \beta=1.1$; the simulation results of the identification method are shown in Figure 6 . The blue dashed line indicates the identification curve of the synchronization parameter observer (SPO), and the red solid line represents 


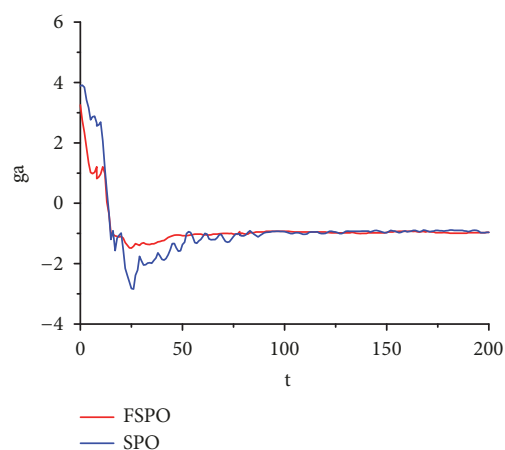

(a)

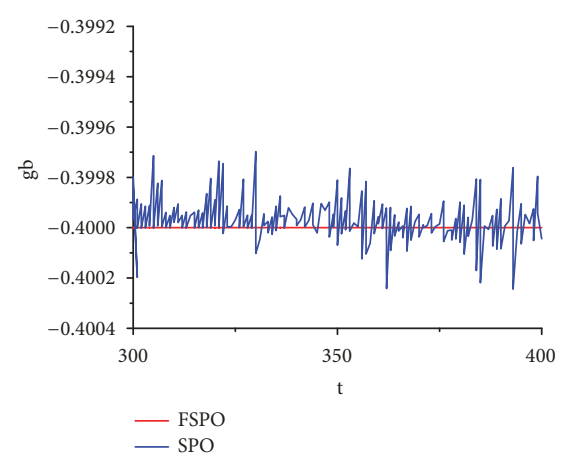

(d)

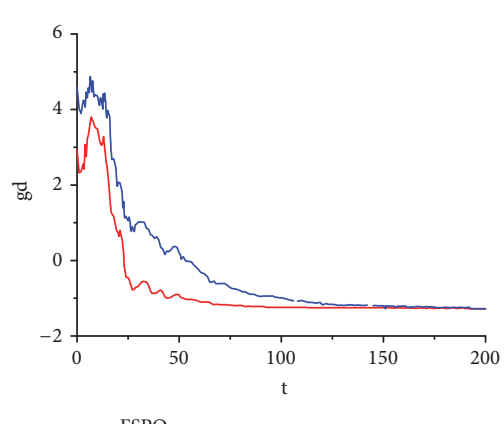

- FSPO

$$
\text { - FSPO }
$$

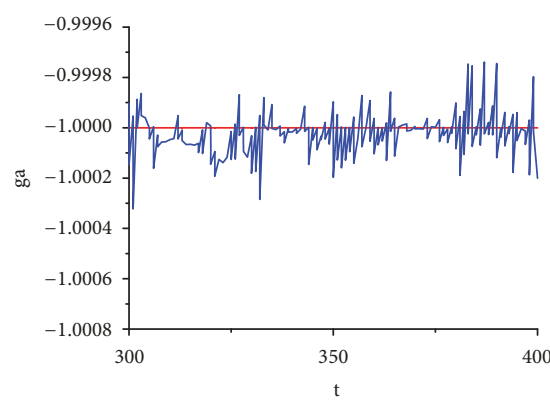

(b)

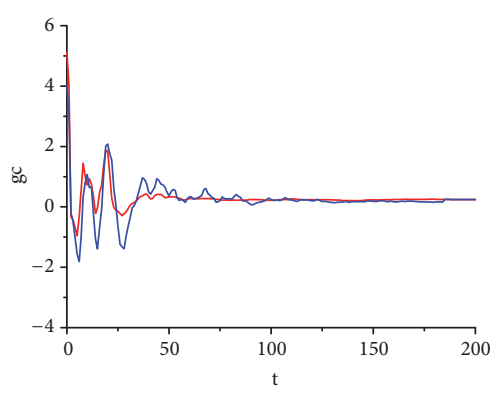

$$
\begin{array}{ll}
{ }_{\mathrm{SSPO}}^{\mathrm{SPO}} & -{ }_{\mathrm{SPO}}^{\mathrm{SPO}}
\end{array}
$$

(e)

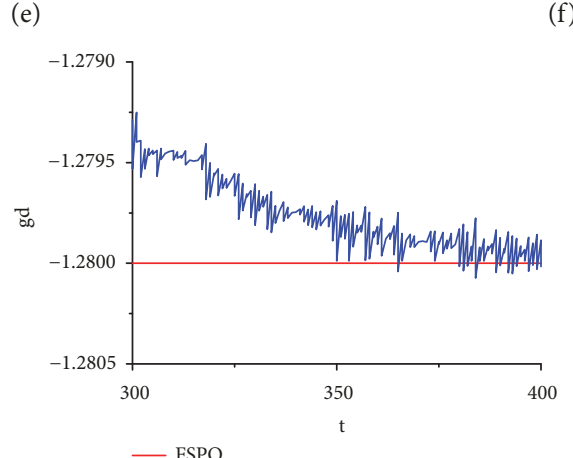

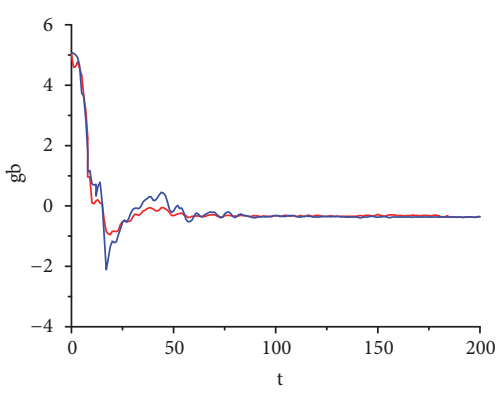

- FSPO

(c)

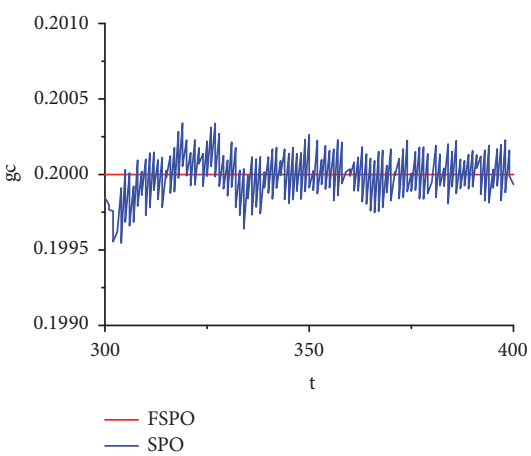

(f)

(g)

(h)

Figure 6: Identifications of the uncertain parameters $a, b, c$ and $d$. (a) The identification of parameter a. (b) The partial magnification of Figure (a). (c) The identification of parameter b. (d) The partial magnification of Figure (c). (e) The identification of parameter c. (f) The partial magnification of Figure (e). (g) The identification of parameter d. (h) The partial magnification of Figure (g).

the identification curve of the fixed-time synchronization parameter observer (FSPO).

To measure the dynamic and static performance of the identification system, we define $\pm 3 \%$ of the identified target value to identify the stable area. As shown in the experimental simulation, we observe that both methods can accurately identify the parameters to the target value of $a=-1, b=-0.4$, $c=0.2, d=-1.28$.

There are obvious differences between the rapidity and stability of identification. The parameters $\mathrm{a}, \mathrm{b}, \mathrm{c}$, and $\mathrm{d}$ are driven to the target value for a long time; the overshoot of identification curve is large with obvious chattering phenomenon in the stable region by SPO. However, the target value of FSPO approach is faster, and the overshoot of identification curve is less with no obvious chattering phenomenon basically. The data of adjustment time and absolute error in the stable area is shown in Table 1.

In summary, compared with the parameter identification based on synchronous observer, the FSPO has the following advantages:

The speed of identification is accelerated and the stability of the system is improved without obvious overshoot and chattering phenomenon. Multiple Object synchronous identification is realized, and the dynamic and static performance of the identification system are good. But the parameter identification time is still long, so we are studying more superior methods to optimize parameter identification. 
TABLE 1: Performance comparison of SPO and FSPO.

\begin{tabular}{lcccc}
\hline \multirow{2}{*}{ Identification parameters } & \multicolumn{2}{c}{ Adjustment time t/s } & \multicolumn{2}{c}{ Absolute Error } \\
& SPO & FSPO & SP0 & $0.06 \%$ \\
a & 128.02 & 72.50 & $0.10 \%$ & $<0.01 \%$ \\
$\mathrm{~b}$ & 157.41 & 86.97 & $0.10 \%$ & $<0.01 \%$ \\
$\mathrm{c}$ & 193.92 & 94.88 & $0.10 \%$ & $<0.01 \%$ \\
$\mathrm{~d}$ & 166.44 & 85.73 & & $<0.01 \%$ \\
\hline
\end{tabular}

\section{Conclusions}

In this paper, we have studied the adaptive fixed-time stability of chaotic oscillation in second order power system. An efficient adaptive controller is proposed, which can stabilize the chaotic oscillation in second order power system in fixed time. Then the uncertain parameters can be identified in fixed time by synchronous observer with adaptive law of parameters. The simulation results show the effectiveness and feasibility of the proposed method. In the high-order systems with interference, the convergence time of the proposed method may be slow, but it is still superior to other methods. The improved method is being studied to solve this problem by us. In addition, considering that noise perturbation is ubiquitous, we will investigate a methodology to solve noise perturbation in our future work and further research the fixed-time stability of fourth-order power system.

\section{Data Availability}

The data used to support the findings of this study are available from the corresponding author upon request.

\section{Conflicts of Interest}

The authors declare no conflicts of interest.

\section{Authors' Contributions}

Caoyuan Ma and Wenbei Wu contributed equally to this work and should be considered co-first authors.

\section{Acknowledgments}

This work is supported by the National Natural Science Foundation of China (71573256) and National Key Research and Development Plan of China (2017YFC0804408).

\section{References}

[1] N. Kopell and J. Washburn, "Chaotic motions in the twodegree-of-freedom swing equations," Institute of Electrical and Electronics Engineers Transactions on Circuits and Systems, vol. 29, no. 11, pp. 738-746, 1982.

[2] E. Ott, C. Grebogi, and J. A. Yorke, "Controlling chaos," Physical Review Letters, vol. 64, no. 11, pp. 1196-1199, 1990.

[3] B. H. Wang, Q. Zhang, C. W. Yang, and W. Yang, Chaotic oscillation control of electric power system based on adaptive backstepping, Electric Power Automation Equipment,
[4] H. O. Wang, E. H. Abed, and A. M. A. Hamdan, "Bifurcations, chaos, and crises in voltage collapse of a model power system," IEEE Transactions on Circuits and Systems I: Fundamental Theory and Applications, vol. 41, no. 4, pp. 294-302, 1994.

[5] X. Chen, W. Zhang, and W. Zhang, "Chaotic and Subharmonic Oscillations of a Nonlinear Power System," IEEE Transactions on Circuits and Systems II: Express Briefs, vol. 52, no. 12, pp. 811815, 2005.

[6] F. Min, Y. Wang, G. Peng, E. Wang, and J. A. Auth, "Bifurcations, chaos and adaptive backstepping sliding mode control of a power system with excitation limitation," AIP Advances, vol. 6, no. 8, 2016.

[7] M. M. Zirkohi, T. Kumbasar, and T.-C. Lin, "Hybrid adaptive type-2 fuzzy tracking control of chaotic oscillation damping of power systems," Asian Journal of Control, vol. 19, no. 3, pp. 11141125, 2017.

[8] C. P. Uzunoglu, Y. Babacan, F. Kacar, and M. Ugur, "Modeling and Suppression of Chaotic Ferroresonance in a Power System by Using Memristor-based System," Electric Power Components and Systems, vol. 44, no. 6, pp. 638-645, 2016.

[9] X. Li and C. A. Cañizares, "Chaotic behavior observations in a power system model," in Proceedings of the IEEE Bucharest PowerTech: Innovative Ideas Toward the Electrical Grid of the Future, Bucharest, Romania, July 2009.

[10] D. Huang, “Adaptive-feedback control algorithm," Physical Review E: Statistical, Nonlinear, and Soft Matter Physics, vol. 73, no. 6, 2006.

[11] H. Mokayed and A. H. Mohamed, "A robust thresholding technique for generic structured document classifier using ordinal structure fuzzy logic," International Journal of Innovative Computing, Information and Control, vol. 10, no. 4, pp. 15431554, 2014.

[12] H. Zhao, Y. Ma, S. Liu, and Y. Yue, "Fuzzy sliding mode variable structure control of chaotic power system with uncertainty," Journal of Computational Information Systems, vol. 7, no. 6, pp. 1959-1966, 2011.

[13] L. Yuan, K.-Y. Wei, B.-X. Hu, and N. Wang, "Nonsingular terminal sliding-mode controller with nonlinear disturbance observer for chaotic oscillation in power system," in Proceedings of the 35th Chinese Control Conference, CCC 2016, pp. 33163320, July 2016.

[14] Y. Sun, L. Zhao, M. Liu, W. Weng, and Q. Meng, "Finite-time leader-following consensus problem of multi-agent systems," Bjog An International Journal of Obstetrics \& Gynaecology, vol. 119, no. 1, pp. 6043-6046, 2012.

[15] Y. Sun, F. Liu, W. Li, and H. Shi, "Finite-time flocking of CuckerSmale systems," in Proceedings of the 34th Chinese Control Conference, CCC 2015, pp. 7016-7020, China, July 2015.

[16] Y. Dong and F. Yang, "Finite-time stability and boundedness of switched nonlinear time-delay systems under state-dependent switching," Complexity, vol. 21, no. 2, pp. 267-275, 2015. 
[17] T. Jing and F. Chen, "Finite-time lag synchronization of delayed neural networks via periodically intermittent control," Complexity, vol. 21, no. S1, pp. 211-219, 2016.

[18] K. Mathiyalagan and K. Balachandran, "Finite-time stability of fractional-order stochastic singular systems with time delay and white noise," Complexity, vol. 21, no. S2, pp. 370-379, 2016.

[19] H. Zhao, Y.-J. Ma, S.-J. Liu, S.-G. Gao, and D. Zhong, "Controlling chaos in power system based on finite-time stability theory," Chinese Physics B, vol. 20, no. 12, Article ID 120501, 2011.

[20] N. Cai, W. Li, and Y. Jing, "Finite-time generalized synchronization of chaotic systems with different order," Nonlinear Dynamics, vol. 64, no. 4, pp. 385-393, 2011.

[21] A. Polyakov, "Nonlinear feedback design for fixed-time stabilization of linear control systems," IEEE Transactions on Automatic Control, vol. 57, no. 8, pp. 2106-2110, 2012.

[22] J. Ni, L. Liu, C. Liu, X. Hu, and T. Shen, "Fixed-time dynamic surface high-order sliding mode control for chaotic oscillation in power system," Nonlinear Dynamics, vol. 86, no. 1, pp. 401420, 2016.

[23] J. D. Cao and R. X. Li, "Fixed-time synchronization of delayed memristor-based recurrent neural networks," Science China Information Sciences, vol. 60, no. 3, Article ID 032201, 2017.

[24] J. Fu and J. Wang, "Fixed-time coordinated tracking for secondorder multi-agent systems with bounded input uncertainties," Systems \& Control Letters, vol. 93, pp. 1-12, 2016.

[25] S. E. Parsegov, A. E. Polyakov, and P. S. Shcherbakov, "Fixedtime consensus algorithm for multi-agent systems with integrator dynamics," in Proceedings of the 4th IFAC Workshop on Distributed Estimation and Control in Networked Systems, NecSys 2013, pp. 110-115, September 2013.

[26] A. Polyakov, D. Efimov, and W. Perruquetti, "Finite-time and fixed-time stabilization: Implicit Lyapunov function approach," Automatica, vol. 51, pp. 332-340, 2015.

[27] D. Q. Wei and X. S. Luo, "Passivity-based adaptive control of chaotic oscillations in power system," Chaos, Solitons \& Fractals, vol. 31, no. 3, pp. 665-671, 2007.

[28] Q. Zhang and B.-H. Wang, "Controlling power system chaotic oscillation by time-delayed feedback," Power System Technology, vol. 28, no. 7, pp. 23-26, 2004.

[29] Y. Sun, X. Wu, L. Bai, Z. Wei, and G. Sun, "Finite-time synchronization control and parameter identification of uncertain permanent magnet synchronous motor," Neurocomputing, 2015.

[30] H. K. Khalil, Nonlinear systems, Prentice-Hall, Inc, Upper Saddle River, NJ, 3rd edition, 2002.

[31] S. M. Wang, C. Y. Yue, and H. G. Luo, "Identification of parameters in Liu's chaotic systems using unknown parameter observers," Journal of Huazhong University of Science and Technology. Natural Science Edition. Huazhong Keji Daxue Xuebao. Ziran Kexue Ban, vol. 35, no. 6, pp. 47-49, 2007. 


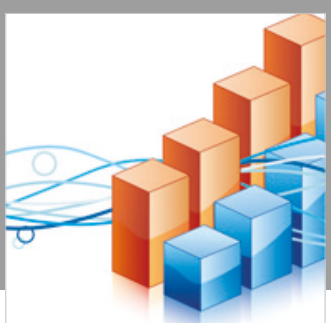

Advances in

Operations Research

\section{-n-m}
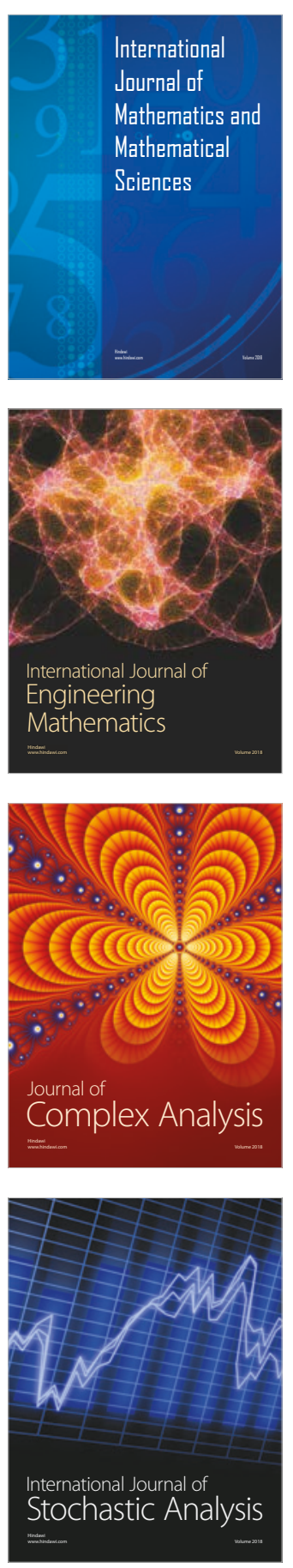
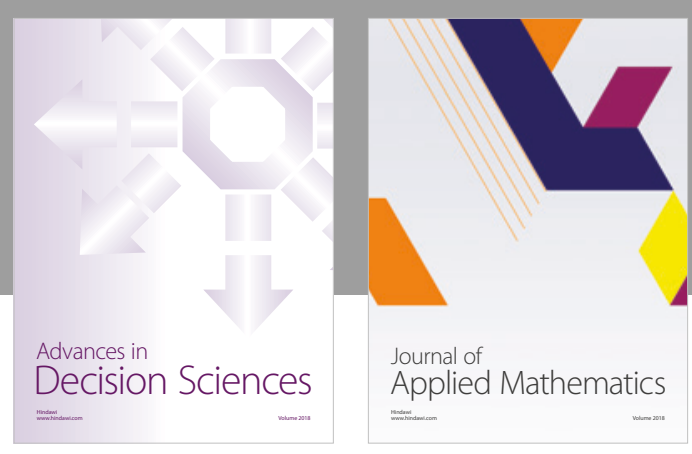

Journal of

Applied Mathematics
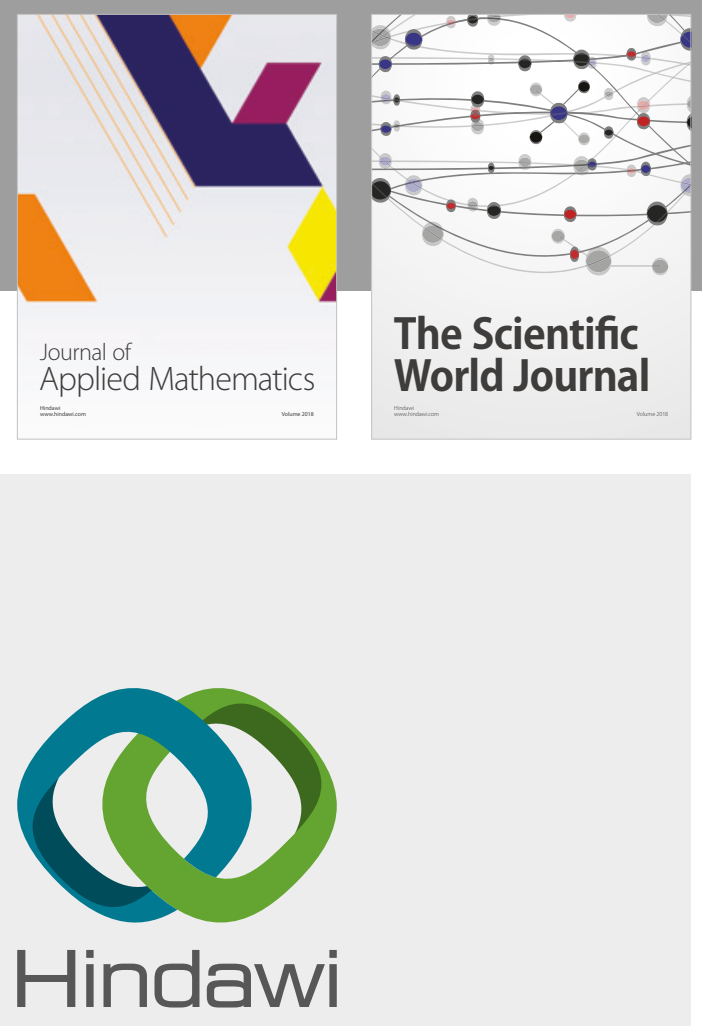

Submit your manuscripts at

www.hindawi.com

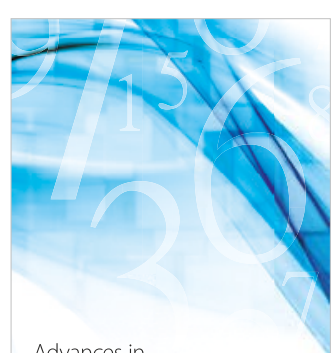

Advances in
Numerical Analysis
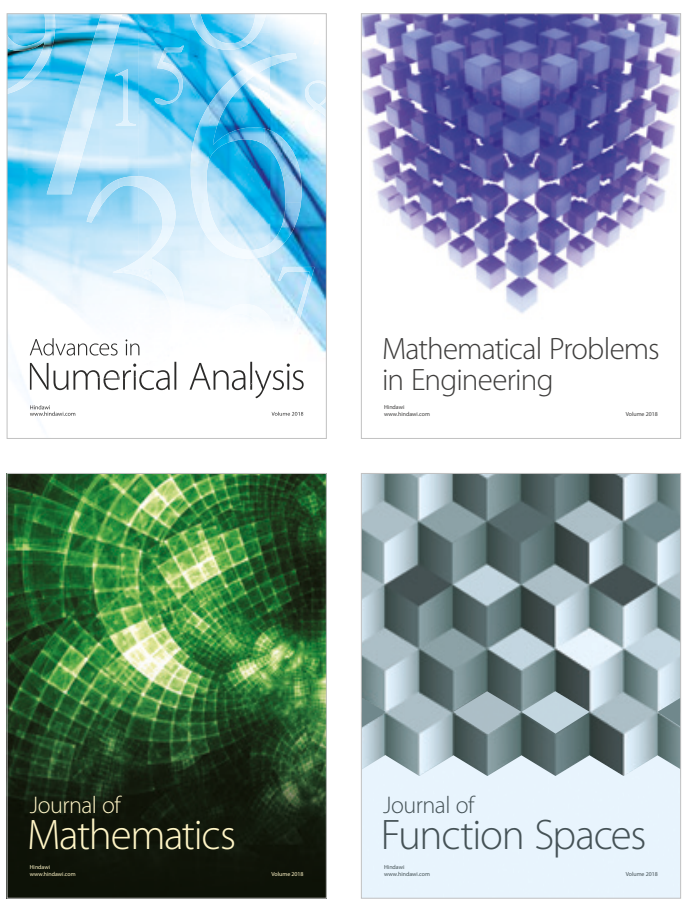

Mathematical Problems in Engineering

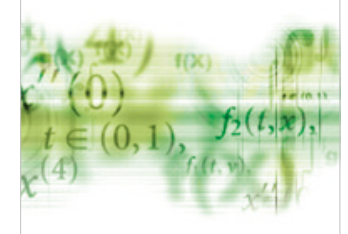

International Journal of

Differential Equations

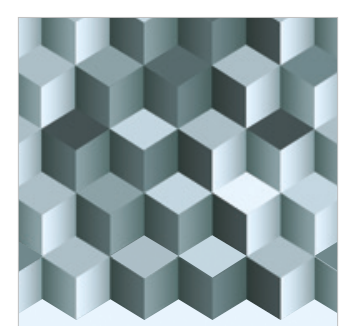

Journal of

Function Spaces

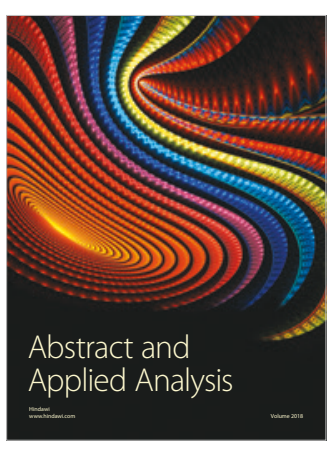

The Scientific

World Journal

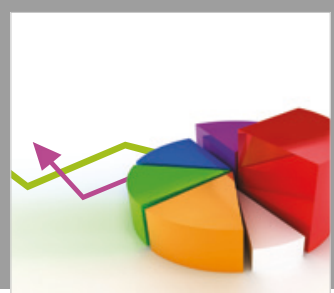

Journal of

Probability and Statistics
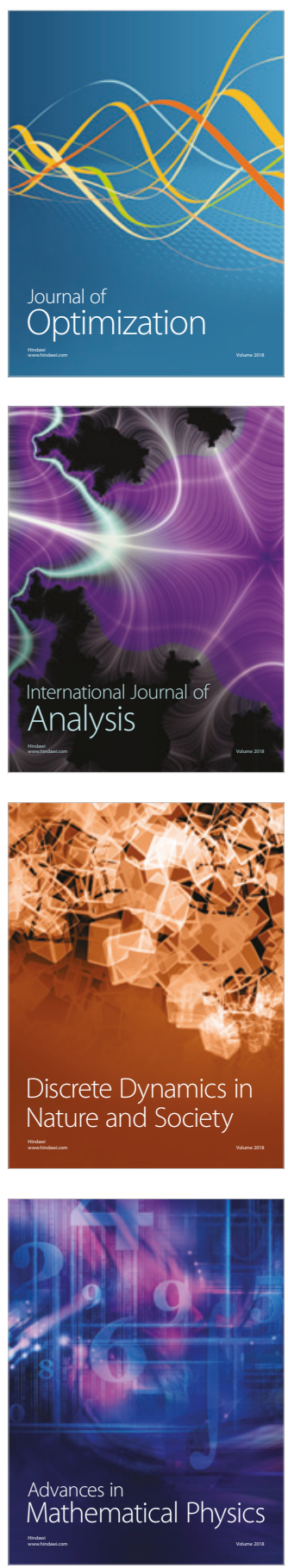